\title{
Abiraterone Acetate: Targeting Persistent Androgen Dependence in Castration-Resistant Prostate Cancer
}

\author{
Lauren C. Harshman • Mary-Ellen Taplin
}

To view enhanced content go to www.advancesintherapy.com

Received: April 5, 2013 / Published online: August 27, 2013

(C) The Author(s) 2013. This article is published with open access at Springerlink.com

\section{ABSTRACT}

Abiraterone acetate is the first second-line hormonal agent proven to improve survival in metastatic castration-resistant prostate cancer. It selectively inhibits cytochrome P450 17 (CYP17) $\alpha$-hydroxylase and cytochrome ${ }_{17,20}$ $\left(\mathrm{C}_{17,20}\right)$-lyase, which are enzymes critical for androgen synthesis. Abiraterone acetate was initially approved in the United States in 2011 after demonstrating a 4-month survival benefit in docetaxel-refractory metastatic prostate cancer. The FDA recently expanded its indication for use in the pre-chemotherapy setting after it elicited significant delays in disease progression and a strong trend for increased overall survival in phase III studies. Ongoing investigations of abiraterone are

L. C. Harshman $(\varangle) \cdot$ M.-E. Taplin

Dana-Farber Cancer Institute, 450 Brookline Ave,

DANA 1230, Boston, MA 02215, USA

e-mail: laurenc_harshman@dfci.harvard.edu

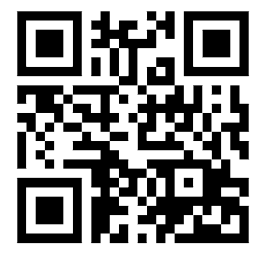

Enhanced content for Advances in Therapy articles is available on the journal web site: www.advancesintherapy.com evaluating its efficacy in earlier disease states, exploring its synergy in combination with other therapeutic agents, and assessing the necessity for administration of concurrent steroids and gonadal suppression. The identification and development of predictive biomarkers will optimize the incorporation of abiraterone into the management of advanced prostate cancer.

Keywords: Abiraterone;

Androgen dependence; Castration-resistant prostate cancer; CYP17 inhibitor; Oncology; Prostate cancer; Survival

\section{INTRODUCTION}

The last 3 years have witnessed tremendous advances in the treatment of castrationresistant prostate cancer (CRPC), a disease from which approximately 30,000 men die annually in the United States [1]. After a 6-year dearth of survival-improving therapies, five agents have emerged that improve survival in CRPC. In 2010, the first cancer vaccine, sipuleucel-T, and a second-generation taxane, cabazitaxel, were approved [2, 3]. 2011 brought 
abiraterone acetate (Zytiga $^{\circledR}$, Janssen Biotech, Inc, Horsham, PA, USA), a first-in-class, highly potent and tolerable androgen biosynthesis inhibitor, which elicited a median 4-month survival benefit in docetaxel-refractory patients $[4,5] .2012$ showcased the survival-improving abilities of both enzalutamide (formerly MDV3100), an agent engineered to block both the binding of androgens to the androgen receptor (AR) and nuclear transport of $\mathrm{AR}$, and a novel bone metastasis-homing radiotherapeutic agent, radium-223 chloride (formerly Alpharadin ${ }^{\circledR}$, Algeta US, LLC, Cambridge, MA, USA and Bayer, Pittsburgh, PA, USA) [6-8]. Abiraterone acetate moved to the pre-chemotherapy setting after demonstrating improvements in progressionfree survival (PFS) and overall survival (OS) when compared to prednisone/placebo [9]. Finally, the introduction of a novel boneprotective agent, denosumab, which delays time to skeletal metastasis and skeletal-related events in the 'pre-abiraterone/enzalutamide' era, has complemented these survivalenhancing cytotoxic and hormonal therapies $[10,11]$.

Prostate cancer is a very heterogeneous disease, which can range from slowly growing, indolent disease that would never induce death, to aggressive variants that necessitate immediate initiation of systemic therapy to control disease progression and symptoms. Multiple lines of evidence have shown that androgens drive the growth and survival of prostate cancer cells $[12,13]$. The pioneering work of Charles Huggins and colleagues [14] in the 1940s formed the cornerstone of the initial treatment for advanced prostate cancer, which is directed at ablating androgen synthesis pathways either by medical or surgical castration. While initially immensely effective in most patients, within $2-3$ years, almost all patients with metastatic disease develop insensitivity to first-line androgen deprivation therapy (ADT) despite castrate levels of serum testosterone, a disease state now referred to as CRPC or, in the past, hormone-refractory or androgen-independent disease $[15,16]$.

While the expanding treatment armamentarium is encouraging, CRPC remains lethal and the individual survival benefits of even the newest agents remain modest at 2-5 months [2-6, 8, 17]. Further, the taxanes have cumulative toxicities, and the immunotherapeutic sipuleucel-T is labor intensive, and elicits few tangible prostatespecific antigen (PSA) or radiologic responses to decrease patient anxiety and control symptoms [3, 17]. Moreover, all of the new agents are costly. Administration of second-line hormonal therapies is often pursued prior to chemotherapy given their enhanced tolerability and with the rationale of targeting the theoretical persistent dependence on AR signaling, even after castration resistance has been proven clinically. The PSA responses achieved by the adrenal steroid biosynthesis inhibitor, ketoconazole, and second-line antiandrogens, like nilutamide, provide evidence for this continued reliance on androgens in CRPC [18-21]. The more substantial declines in PSA and improvements in survival with abiraterone acetate and enzalutamide validate this principle.

This review will focus on abiraterone acetate, which is a selective inhibitor of cytochrome P450 17 (CYP17) $\alpha$-hydroxylase and cytochrome $_{17,20}\left(\mathrm{C}_{17,20}\right)$-lyase, enzymes, which are critical to androgen synthesis (Fig. 1). Abiraterone acetate elicits significant antitumor activity in CRPC. It induces PSA declines of $\geq 50 \%$ in $29-62 \%$ of patients, achieves OS benefits in both docetaxelrefractory and chemotherapy-naïve patients, 


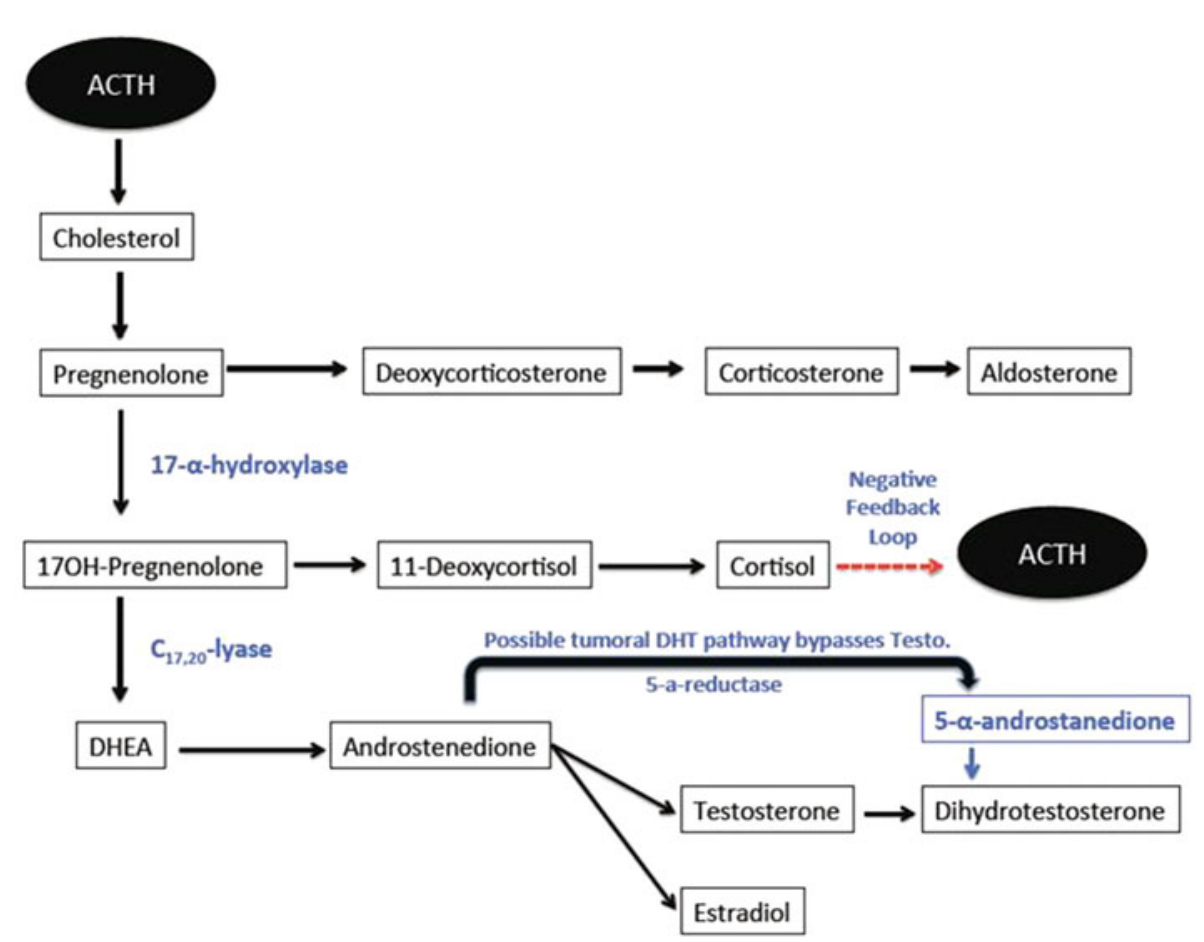

Fig. 1 Androgen axis: physiologic pathways and possible tumor bypass pathway for the production of testosterone. $A C T H$ adrenocorticotropic hormone, DHEA dihydroepiandrostendione, $D H T$ dihydrotestosterone, Testo testosterone

delays and reduces skeletal-related events, and palliates pain $[4,9]$ (Table 1). This multi-faceted efficacy, along with an excellent safety and tolerability profile compared to chemotherapy, positions abiraterone acetate as an agent that enhances patient outcomes and quality of life in the pre-chemotherapy setting, and it may be incorporated as part of novel treatment approaches in earlier disease states. We will detail the development of abiraterone acetate, consider its placement in the expanding CRPC treatment armamentarium, and discuss future strategies to enhance its effectiveness.

\section{CASTRATION-RESISTANT PROSTATE CANCER}

Phase III trials completed in the 1990s reported a median OS of 16-18 months from the start of docetaxel in CRPC. In contrast, contemporary phase III trials of androgen axis inhibitors, such as abiraterone acetate and enzalutamide, given after docetaxel therapy have reported a median survival of 15-18 months [4, 6]. This improvement in median survival is related to both a shift toward earlier initiation of treatment and a real improvement in survival elicited by these new agents.

The drivers behind the progression to CRPC are multifactorial but can be attributed to persistence of androgens from non-gonadal sources and intratumoral AR signaling. Rarer transformation to true androgen axis independence can also occur [22]. In the eugonadal state, the testes synthesize $80 \%$ of the body's testosterone, but another $20 \%$ is produced by the adrenal glands, and in CRPC, the tumor itself can produce intracrine androgens [23]. Tissue studies in healthy volunteers have demonstrated that despite 


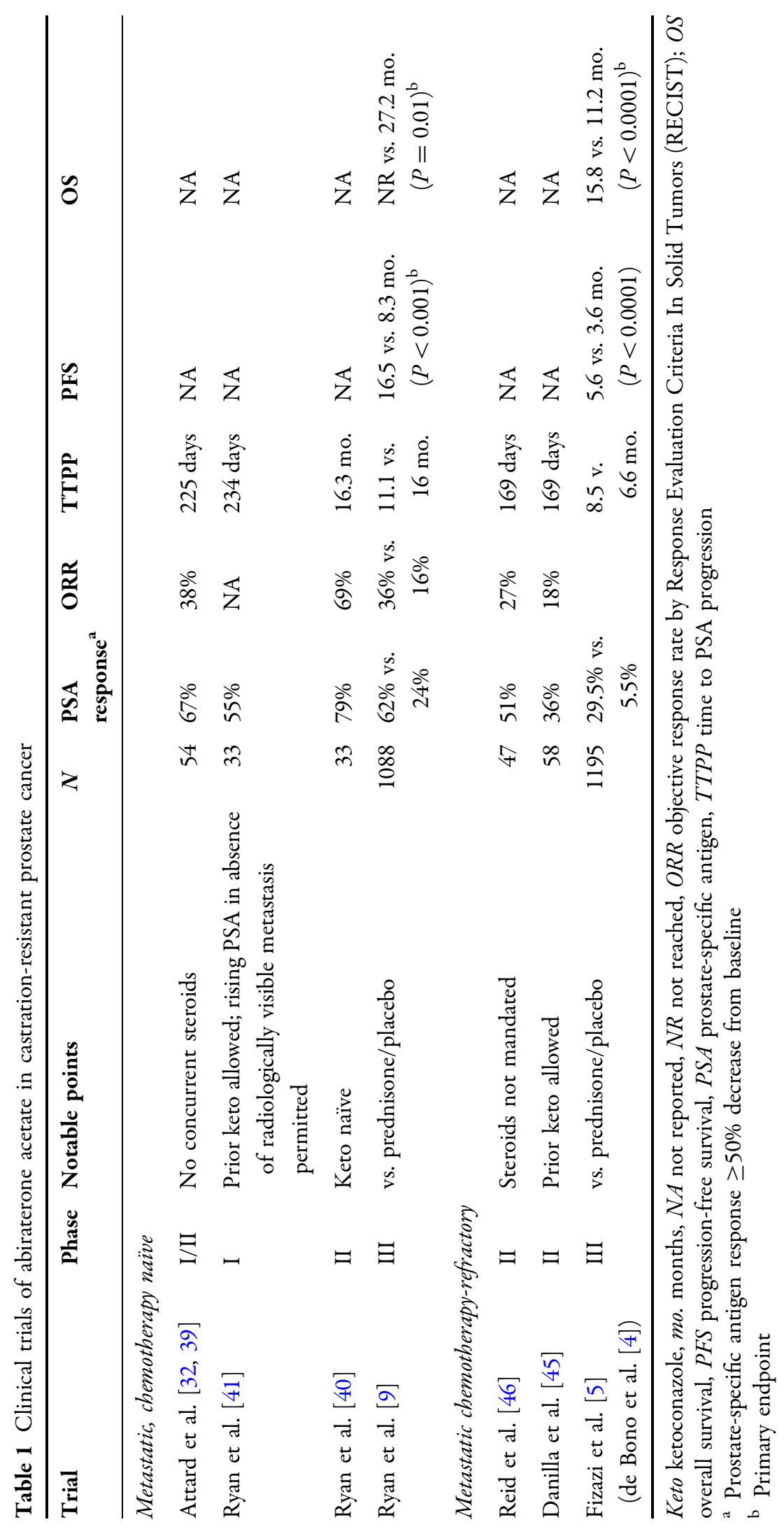


induction of castrate levels of serum testosterone with gonadotropin-releasing hormone (GnRH) agonists, intraprostatic tissue androgen levels may only decline by approximately 30\%, leaving sufficient ligand to stimulate the AR [24]. In hormone-sensitive prostate cancer patients, Mostaghel and colleagues [25] examined the effects of shortterm (1-9 months) castration on intraprostatic androgen levels and the expression of androgen-regulated genes. They found significant heterogeneity between serum and prostate tissue levels of testosterone and other markers of androgen regulation. While castration decreased intraprostatic tissue androgens by $75 \%$, not all androgen-regulated genes, such as TMPRSS2 and PSA, were suppressed [25]. Additionally, these investigators demonstrated that the level of tissue testosterone in metastatic CRPC is significantly higher than levels even from eugonadal prostate controls. The authors concluded that the effects of medical castration, as assessed by serum testosterone, did not correspond with the degree of androgen depletion occurring in the prostate tissue or CRPC metastases [25]. This persistent repository of tumor androgen enables adaptive AR-related resistance mechanisms to develop. Together, these studies bring into question our historical use of serum testosterone as a surrogate marker for tumor androgen activity. Identification of better proxies for tumor androgen depletion and more effective strategies to neutralize nongonadal androgen sources is imperative.

AR-related mechanisms for tumor growth in CRPC include: AR amplification; AR splice variants leading to constitutively active receptors; mutations leading to promiscuous AR activation by weak androgens, other steroid hormones, and even traditional antagonists, such as antiandrogens; changes in AR coregulatory proteins; and synthesis of tumor androgens [26-30]. Increased expression of genes converting adrenal androgens or tissue precursors, such as cholesterol to testosterone, has been observed in CRPC [28]. Tumoral androgen production may even stem from variant synthetic pathways. Work by the Sharifi Laboratory demonstrates that contrary to conventional thought, the dominant route of tumoral dihydrotestosterone (DHT) synthesis in CRPC actually bypasses testosterone and instead, acts through $5 \alpha$-reduction of androstenedione [31].

The AR regulates PSA expression, and an elevated PSA level is usually the first manifestation of CRPC. An elevated PSA may reflect renewed AR activation or ongoing transcription of androgen-AR-regulated genes, which may primarily stimulate or co-stimulate cancer growth [32-34]. This sign of persistent intratumoral AR signaling in CRPC suggests potential sensitivity to more intensive androgen deprivation, which has been evidenced by the $<56 \%$ PSA response rates with older-generation, second-line hormonal agents, such as ketoconazole and the AR antagonists, bicalutamide (daily dose of 50-200 mg), flutamide, and nilutamide [18-20, $35,36]$. The persistence of serum PSA and the responses seen with older, secondary hormonal blockade, combined with a desire to develop less toxic and more selective agents than existing chemotherapeutics has driven the development of the latest generation of $\mathrm{AR}$ and androgen biosynthesis inhibitors. These agents include the ligand synthesis inhibitors, abiraterone acetate and orteronel (TAK-700), the receptor signaling inhibitors, enzalutamide and ARN509, as well as the combined receptor and androgen synthesis inhibitor, galeterone (TOK-001). 


\section{CLINICAL INVESTIGATION OF ABIRATERONE ACETATE IN CRPC}

Abiraterone acetate is the prodrug of abiraterone. It acts through irreversible CYP17 inhibition, but is more targeted and potent than the older generation, non-specific CYP inhibitor, ketoconazole. Its superior efficacy and tolerability reflect this greater selectivity against CYP17, making it significantly more potent than ketoconazole [23, 37]. Abiraterone acetate's inhibition of CYP17 $\alpha$-hydroxylase and $\mathrm{C}_{17,20}$-lyase triggers a cascade of predictable endocrinologic changes (Fig. 1). CYP lyase inhibition results in a reduction of androgens downstream of pregnenolone, including dihydroepiandrostendione

(DHEA), androstenedione, testosterone, and DHT [23]. The decreased conversion of pregnenolone to $17 \mathrm{OH}$-pregnenolone and of progesterone to $17 \alpha-\mathrm{OH}$ progesterone translates into increased levels of pregnenolone and progesterone. However, as 17 OH-pregnenolone and $17-\mathrm{OH}$ progesterone are its substrates, cortisol levels subsequently decrease. The reduction in cortisol activates a negative feedback loop, stimulating adrenocorticotropic hormone (ACTH) production. There is potential for resultant mineralocorticoid excess (ME), which is a possible dose-limiting toxicity of abiraterone acetate in some patients. However, the incidence of $\mathrm{ME}$ is generally abrogated by concomitant administration of prednisone (or dexamethasone) or aldosterone antagonists, such as eplerenone or even spironolactone [38]. The most common symptoms of ME observed with abiraterone acetate administration are hypokalemia, hypertension, and edema, and were seen in 17, 10-22, and $28-31 \%$ of patients, respectively, in the phase III studies $[4,9]$.
In contrast to the prevention of ligand binding that occurs with AR antagonists, or the singular inhibition of gonadal androgen synthesis that occurs with GnRH agonists or antagonists, abiraterone acetate's effects are wide-ranging. All androgen synthesis pathways rely on CYP17; thus, abiraterone acetate disturbs adrenal, gonadal, and intratumoral androgen production [23]. Created by chemists at the Royal Marsden Hospital in the UK, abiraterone acetate's development for the treatment of prostate cancer was delayed for 10 years due to safety concerns at the time over the associated adrenal insufficiency and because of a disinterest in secondary hormonal blockade as a viable therapeutic maneuver for prostate cancer [38]. The investigators, de Bono and Attard [32, 39], resurrected interest in the compound and initiated phase I testing in a group of asymptomatic, chemotherapy-naïve patients with good performance status. The initial studies provided important insight into abiraterone acetate's therapeutic effects and toxicity profile in the absence of concomitant steroids.

Abiraterone acetate was initially studied in the chemotherapy-naïve CRPC setting [32, 3941]. In the first phase I/II trial, de Bono and Attard evaluated abiraterone as monotherapy without concomitant steroids in 54 patients [32]. Continued administration of a luteinizing hormone-releasing hormone (LHRH) agonist was mandated to prevent any compensatory luteinizing hormone (LH) surge and testosterone production that might overwhelm abiraterone acetate's CYP17 blockade [32, 42]. While up to 2,000 $\mathrm{mg}$ per day was found to be tolerable, a plateau in pharmacodynamic effect advocated for $1,000 \mathrm{mg}$ as the recommended monotherapy dose [39]. Notably, abiraterone also induced near-undetectable levels of 
circulating androgens, such as serum testosterone, DHEA-sulfate, and DHT, but increased levels of mineralocorticoids, such as deoxycorticosterone and aldosterone. In fact, the development of exquisitely sensitive assays, such as liquid chromatography tandem mass spectrometry, was required to measure such low levels of androgen [39]. Despite the increased levels of mineralocorticoids, no symptoms of ME were reported. Of the 42 patients enrolled in this phase II trial who were treated with abiraterone acetate $1,000 \mathrm{mg}$ daily, $67 \%$ achieved a PSA decline of $\geq 50 \%$. In the 24 patients with measurable disease by Response Evaluation Criteria in Solid Tumors (RECIST), 38\% experienced partial responses. The median time to PSA progression (TTPP) was 225 days $[95 \%$ confidence interval (CI), 162-287 days]. As per protocol, dexamethasone ( $0.5 \mathrm{mg}$ daily) was added at the time of progression in order to evaluate whether steroids could reverse the possible effects of excess ACTH and upstream steroids. The latter have been implicated in activating a mutated or promiscuous androgen receptor, if present [43, 44]. The addition of dexamethasone resulted in a subsequent PSA decline ranging from 36-99\% in 4 of 15 patients, suggesting re-sensitization to abiraterone acetate. The duration of response to the dexamethasone-abiraterone acetate combination ranged from $\geq 49$ to $>348$ days; all responses were ongoing at the time of publication of the results. Higher pre-treatment androgen and estradiol levels were associated with an increased probability of PSA response and prolonged TTPP [32]. These findings may indicate that these serum hormone levels are predictive biomarkers that could optimize the selection of patients who would be most likely to benefit from abiraterone acetate therapy.

Targeting a somewhat less advanced disease subset, a concurrent phase I trial of abiraterone acetate was performed in 33 patients with progressive CRPC, as evidenced by an elevated PSA level with or without radiologic evidence of distant metastasis [41]. In addition, this trial differentiated itself from the initial Attard trial [39] by enrolling patients who had received prior treatment with ketoconazole. This relaxation of eligibility criteria permitted important data capture on abiraterone acetate's efficacy in a disease state of unmet need-biochemical relapse-and addressed the clinically relevant question of whether past ketoconazole administration conferred resistance to abiraterone acetate, given their similar mechanisms of action through CYP inhibition. However, the limitation of this inclusiveness was a resultant heterogeneous patient population. As expected, the majority (70\%) of patients had bony metastases while only $18 \%$ had visceral metastases [41]. A smaller proportion $(9 \%)$ of patients had evidence of biochemical recurrence, or locally advanced disease with no signs of distant metastasis on imaging. More than half $(58 \%)$ of the patients had received prior ketoconazole with the majority (84\%) having ketoconazole-sensitive disease, as evidenced by PSA declines of $\geq 50 \%$ during ketoconazole administration. Based on data from the Attard study, including clinical responses, maximization of anticipated endocrinologic effects, and its favorable safety profile, dose escalation of abiraterone acetate was stopped at $1,000 \mathrm{mg} / \mathrm{day}$. In the entire cohort, decline of PSA levels at week 12 occurred in $55 \%$ of patients, including nine who had received prior ketoconazole. The median time to PSA progression was 234 days, likely reflecting the earlier disease state of patients compared with those in the Attard trial. Median duration of therapy was 15 months. Responses with abiraterone acetate were observed not only in patients who had not 
responded (33\%) to ketoconazole, but also in those who had initially responded but eventually progressed on ketoconazole (46\%) [41]. These latter results indicate a lack of crossresistance between the two agents.

Confirming the preliminary efficacy observed in the phase I studies, a subsequent phase II trial evaluated abiraterone acetate plus prednisone in 33 patients with progressive, metastatic, CRPC who were both chemotherapy- and ketoconazole-naïve [40]. The great majority (79\%) of patients had a decline in PSA levels of $\geq 50 \%$. Disease control was remarkably durable for this population, with a median time to PSA progression of 16.3 months. Interestingly, discordant bone scans occurred in nearly half (11/23) of the patients who were thought to be responding to abiraterone acetate by PSA criteria [40]. These discordant responses were later confirmed to be flare phenomena, since the lesions improved on subsequent scans. Flare phenomenon occurs when, despite other signs of clinical response such as PSA decline, bone lesions appear more intense on bone scan. The 'worsening' of the lesion actually reflects treatment response, but may be misinterpreted as disease progression by the reviewer [40]. The requirement by Ryan and colleagues for a confirmatory scan highlights the potential for this phenomenon to occur with abiraterone acetate treatment. It may occur in up to $50 \%$ of cases, which emphasizes the importance of confirmatory scans prior to therapy discontinuation with this agent [40]. Evolving imaging techniques with novel tracers and the incorporation of serum and urine markers of bone turnover, such as bonespecific alkaline phosphatase, may further distinguish flare effects due to treatment response from those of disease progression.

The two phase II trials evaluating abiraterone acetate in CRPC reported response rates of
$36-51 \%$ and TTPP of 168 days, and provided the basis for the first phase III study in the docetaxel-refractory CRPC setting $[45,46]$. The primary endpoint of the first phase III study was OS. Secondary endpoints included time to PSA progression, PFS according to radiologic findings based on pre-specified criteria, and the PSA response rate [4]. Patients were randomized in a 2:1 fashion to receive either abiraterone acetate/prednisone (oral abiraterone $1,000 \mathrm{mg}$ once daily in combination with oral prednisone $5 \mathrm{mg}$ twice daily, $n=797$ ) or placebo/prednisone (oral placebo once daily in combination with oral prednisone $5 \mathrm{mg}$ twice daily, $n=398$ ). The majority of patients had an Eastern Cooperative Oncology Group (ECOG) performance status of 0 or 1 . Ninety percent of patients had bony metastases and 30\% had visceral involvement. While all patients had received at least one line of docetaxel therapy, approximately $30 \%$ of patients had received two prior lines of chemotherapy. Patients who had received prior ketoconazole therapy for prostate cancer, or had neuroendocrine differentiation of their prostate cancer were excluded [4].

At the time of interim analysis, abiraterone acetate/prednisone induced significantly longer OS compared to prednisone/placebo [14.8 vs. 10.9 months, respectively, hazard ratio (HR) 0.65; 95\% CI 0.54-0.77; $P<0.001$ ] [4]. Median follow-up was 12.8 months. All secondary endpoints favored the abiraterone acetate arm, including time to PSA progression (10.2 vs. 6.6 months, respectively; $P<0.001$ ), PFS (5.6 vs. 3.6 months, respectively; $P<0.001$ ), and PSA response rate $(29 \%$ vs. $6 \%$, respectively; $P<0.001)$. The final analysis of the study occurred before unblinding and subsequent patient crossover from placebo to abiraterone acetate [5]. After 775 of the pre-specified 799 death events and a median 20.2 months of 
follow-up, median OS was 15.8 months versus 11.2 months for the abiraterone arm compared with the placebo arm, respectively (HR 0.74, 95\% CI 0.64-0.86; $P<0.0001$ ). Median time to PSA progression was 8.5 months, median radiologic PFS was 5.6 months, and $29.5 \%$ of patients had a PSA response on the abiraterone acetate arm; all were statistically significantly increased compared with the corresponding results in the placebo arm, and were similar to the initial efficacy analysis [5].

The most common toxicities attributed to abiraterone acetate in the phase III, postdocetaxel study were related to testosterone depletion and $\mathrm{ME}$, such as fluid retention, hypertension, and hypokalemia. The most common grade 3-4 events occurred with similar frequency in both cohorts: fatigue (9-10\%), anemia (8\%), back pain (7\% vs. $10 \%$ ), and bone pain (6\% vs. $8 \%$ ) [5]. No new safety concerns were identified in the phase III study. Importantly, fewer patients discontinued treatment because of toxicity in the abiraterone acetate group compared to the control group (13\% vs. $18 \%$, respectively) [5].

The phase III, post-docetaxel abiraterone acetate trial was also notable for its integrated correlative assessments of quality of life, fatigue, and pain. These factors are important considerations, as patients with CRPC can range from being asymptomatic to having significant debilitating bony pain or multifactorial fatigue related to anemia, past anti-cancer treatment, or cancer progression. Sternberg and colleagues incorporated into the phase III abiraterone study 'The Brief Fatigue Inventory', a validated fatigue assessment tool as an outcome measure [47]. In patients who reported clinically significant fatigue at study initiation, abiraterone acetate achieved clinically meaningful benefits compared to prednisone alone. It elicited significant reductions in intensity of fatigue, improved fatigue interference, and faster reduction in fatigue intensity [47]. To assess whether pain and skeletal-related events were reduced with abiraterone acetate, Logothetis and colleagues [48] issued a 'Brief Pain Inventory' questionnaire at baseline, day 15 of cycle 1 , and day 1 of each cycle thereafter. In patients with substantial pain at baseline, abiraterone acetate achieved significantly greater improvements in pain palliation, faster time to pain reduction, and interference with daily activities than steroids alone. Abiraterone acetate meaningfully impacted skeletal-related events, defined as pathologic fracture, spinal cord compression, need for palliative radiation to bone, or surgery to the bone. In the entire study population, abiraterone acetate prolonged the median time to occurrence of first skeletal-related event by 5 months (25 vs. 20 months, $P=0.001$ ) [48].

Based on a 4-month survival benefit, the FDA granted approval of abiraterone acetate in April 2011 [4]. Given the need to improve tolerability of therapeutic agents and preference to avoid chemotherapy, abiraterone acetate's outcomes in chemotherapy-naïve patients were highly anticipated. Ryan and colleagues executed a phase III study in asymptomatic or only mildly symptomatic patients with progressive, chemotherapy-naïve metastatic CRPC disease [9]. This multicenter, international trial randomized 1,088 patients in a 1:1 fashion to receive either abiraterone acetate $1,000 \mathrm{mg}$ daily in combination with oral prednisone $5 \mathrm{mg}$ twice daily $(n=546)$ or oral placebo with prednisone $5 \mathrm{mg}$ twice daily $(n=542)$. The coprimary endpoints were radiographic PFS and OS. Secondary endpoints included time to opiate use for cancer-related pain, time to initiation of chemotherapy, time to deterioration in ECOG performance status, 
and time to PSA progression [9]. A modified version of the Prostate Cancer Working Group 2 criteria was used to define progressive disease on bone scan [49]. This trial was noteworthy for being the first phase III study in prostate cancer since the mitoxantrone trials in the late 1990s in which the FDA allowed a primary endpoint other than OS, and may pave the way to testing new compounds at earlier disease stages [50, 51].

In the pre-docetaxel, abiraterone acetate phase III trial, the two arms were well balanced in terms of median age, time from diagnosis, baseline PSA, testosterone, levels of alkaline phosphatase, hemoglobin and lactate dehydrogenase (LDH), and the extent of bone and visceral disease as well as baseline pain [52]. The median duration of follow-up was 22.2 months. The study was halted early after a statistically significant improvement in the primary endpoint of radiographic PFS (rPFS) was observed at the second interim analysis. The median PFS had not been reached in the abiraterone acetate arm but was 8.3 months in the placebo arm (HR 0.43; 95\% CI 0.35-0.52; $P<0.0001$ ) [52]. At the subsequent analysis, the combination of abiraterone acetate-prednisone elicited a median PFS of 16.5 months compared to 8.3 months with prednisone alone (HR 0.53; 95\% CI 0.45-0.62; $P<0.001$ ) [9]. While it did not cross the pre-specified efficacy boundary, there was a robust trend to improvement in OS with abiraterone acetate-prednisone compared to prednisone alone (median not reached vs. 27.2 months; HR 0.75 ; $95 \%$ CI $0.61-0.93$; $P=0.01$ ). Subgroup analyses of baseline performance status, pain level, presence of bone metastasis, age, PSA, LDH and alkaline phosphatase levels, and region all favored an rPFS and OS benefit for treatment with abiraterone acetate [9]. rPFS positively correlated with OS (correlation coefficient
0.72). This relatively strong correlation suggests that rPFS may be a good surrogate for OS in patients receiving abiraterone acetate, but this requires further validation.

Statistically significant PSA declines of $\geq 50 \%$ were elicited in $62 \%$ of patients on abiraterone acetate compared to $24 \%$ of patients on prednisone, and objective RECIST responses in $36 \%$ vs. $16 \%$ of patients in the study arms, respectively [9]. Although significantly lower, the $24 \%$ rate of PSA declines of $\geq 50 \%$, and the objective response rate of $16 \%$ with prednisone alone confirms its single-agent activity. All secondary endpoints, such as time to the need for opiate use for cancer-related pain (not reached vs. 23.7 months) and time to the initiation of cytotoxic chemotherapy (25.2 months vs. 16.8 months) were statistically significantly improved with abiraterone acetate compared with prednisone. Patient-reported outcomes also favored the investigational agent. The statistically significant delays in disease progression, strong trend towards increased OS, the lack of any concerning new toxicities, and uniformly positive secondary endpoints all favored abiraterone acetate's extended approval and use in CRPC patients who have not received prior chemotherapy [9]. Ultimately, on December 10, 2012, the FDA expanded abiraterone acetate's indication to include chemotherapy-naïve CRPC patients [53]. While not reported in Ryan's published manuscript, the FDA announcement conveyed that the median OS for those in the abiraterone acetate arm was 35.3 months compared with 30.1 months for those receiving placebo (HR 0.79; 95\% CI 0.66-0.96) [53]. Despite what we assert is a clinically significant 5-month benefit for abiraterone acetate, this difference was not statistically significant according to the prespecified O'Brien-Fleming boundary of 
$P \leq 0.001$ [53]. The results of this trial and its approval are historic as no previous noncytotoxic, non-chemotherapy agent has significantly impacted PFS or OS in chemotherapy-naïve CRPC patients while maintaining or improving quality of life for our patients.

Importantly, given the relatively fit and often asymptomatic population, no new safety signals were seen despite the longer duration of administration in this study (median 16 months) compared to the registration trial in docetaxel-refractory CRPC (median 8 months; A. Molina, Johnson and Johnson, personal communication). The frequency of treatment discontinuation due to drug toxicity was similar in the two arms. The majority halted study treatment for progressive disease, while only $7 \%$ were discontinued due to toxicity in the abiraterone acetate arm, and 5\% in the placebo arm [52]. Abiraterone acetate induced a higher rate of all grade fatigue (39\%), arthralgias (28\%), peripheral edema (28\%), and hepatotoxicity (11-12\%; $<1 \%$ grade 3 or more) than prednisone [9]. As expected, other side effects related to ME, such as hypertension (22\%) and hypokalemia (17\%), were more common with abiraterone acetate than with prednisone; most were low grade. Hepatotoxicity generally occurred within the first 3 months of treatment and did not contribute to any deaths. Non-hypertensive cardiac events, of which there was a slight increase in the abiraterone acetate arm compared with the prednisone arm (19\% vs. $16 \%$, respectively; grade 3 or $4: 6 \%$ vs. $3 \%$, respectively), tended to have a later onset ( $>3$ months). These incidents included myocardial infarction, heart failure, and arrhythmias. Finally, as per the FDA notice, adrenal insufficiency was observed in $0.5 \%$ of patients on abiraterone acetate vs. $0.2 \%$ of patients on placebo [53].
In summary, clinicians should be vigilant of the rare but real possibility of developing adrenal insufficiency with abiraterone acetate treatment, and the more common and easily treatable symptoms of ME. The long-term toxicities of steroid use, which are especially germane in a longer-living, chemotherapynaïve population, are also an important consideration. While prednisone $5 \mathrm{mg}$ twice daily is the standard regimen used with abiraterone acetate and studied in the phase III setting, de Bono and colleagues initially favored dexamethasone $0.5 \mathrm{mg}$ once daily due to its long half-life and lack of mineralocortical effects, and used it with satisfactory results [32]. There has been no investigation into which patients actually need steroids and at what dosage to prevent ME. Studies are ongoing to determine whether lower doses of steroid, or administration of steroids or an aldosterone antagonist at first signs of toxicity achieve similar effectiveness while optimizing safety and quality of life. Likewise, the need for concurrent $\mathrm{GnRH}$ axis inhibition to prevent a potential LH surge is being investigated; LH surge was observed in two of three non-castrate males given abiraterone, although it is not known whether this effect would be observed in older men with a history of long-term castration [42].

\section{Predictive Biomarkers \\ and Pharmacodynamic Effects on Tissue}

In addition to evaluating their effects on serum androgen and steroid levels, several investigators have attempted to look at the pharmacodynamic consequences of drugs in tumor tissue in order to better elucidate mechanisms of drug response and patterns of resistance, and to identify predictive signatures. In an informative translational study of 
57 patients with bone-metastatic CRPC treated with abiraterone acetate, Efstathiou and colleagues [54] measured androgen signaling in bone marrow-infiltrating CRPC, as well as testosterone levels in serum and marrow aspirate, and correlated these endpoints with clinical outcome. Patients underwent transiliac bone marrow aspirate and biopsies prior to starting treatment with standard-dose abiraterone acetate, at week 8 on therapy, and at the end of this observational study. Corresponding blood plasma and serum levels were collected within $2 \mathrm{~h}$ of the biopsy. Immunohistochemistry (IHC) was used to assess AR and CYP17 expression. Tumorinfiltrating bone marrow samples were obtained in 27 patients at baseline and in 30 patients at any time point. A quarter of patients (14/56) had primary drug-resistant disease [54]. Testosterone concentrations in the marrow aspirate were higher than in blood in 7/42 patients. This study ultimately confirmed that marrow aspirate androgen depletion was generally achieved by 8 weeks, and remained depleted at cessation of therapy. A strong correlation existed between pre-treatment circulating levels and microenvironment (nontissue) marrow aspirate testosterone levels (Pearson's $r=0.91$ ) [54].

The evaluation of nuclear $\mathrm{AR}$ and cytoplasmic CYP17 expression in this work unveiled a potential predictive signature for abiraterone acetate response [54]. AR expression by IHC was frequently higher at baseline than on treatment, while pre-treatment CYP17 expression was more heterogeneous. Homogenous intense expression of nuclear AR in combination with $\geq 10 \%$ CYP17 tumor expression correlated with a longer time to treatment discontinuation ( $>4$ months, $P<0.001)$ [54]. In the cohort of 25 patients whose tumors met these criteria, only one patient had primary drug-resistant disease. Conversely, only two patients whose tumor did not have this expression signature had a long-term response. One patient whose tumor lacked one or both of these findings had primary resistant disease $(n=10)$, indicating that patients without this molecular profile may not benefit from abiraterone acetate [54]. Higher pre-treatment CYP17 tumor expression also correlated with increased testosterone expression in the marrow aspirate, suggesting that these tumors are capable of intratumoral androgen synthesis, which may drive their disease resistance [54]. This work establishes the feasibility and potential value of sampling bone metastasis to explore mechanisms of resistance and to demonstrate that drugs are achieving their on-target effects. It further substantiates that persistent androgen signaling in CRPC tumors is functionally significant and confirms that abiraterone acetate effectively depletes testosterone in blood and bone marrow aspirates. This study validated Attard and colleagues' findings [39] that abiraterone acetate reduced plasma testosterone and DHT levels to undetectable levels, but was the first to prospectively establish that it also reduced marrow aspirate testosterone and DHT concentrations to lessthan-picogram-per-milliliter amounts, and that the levels remained suppressed at progression.

Men with localized but high-risk prostate cancer have suboptimal cure rates with prostatectomy [55]. Neoadjuvant trials of agents, such as abiraterone acetate, that can achieve intense androgen deprivation are a logical approach to improve outcomes in these men and permit pharmacodynamic investigation of the mechanisms of response and resistance. Taplin and colleagues executed the first randomized, neoadjuvant study investigating abiraterone acetate's 


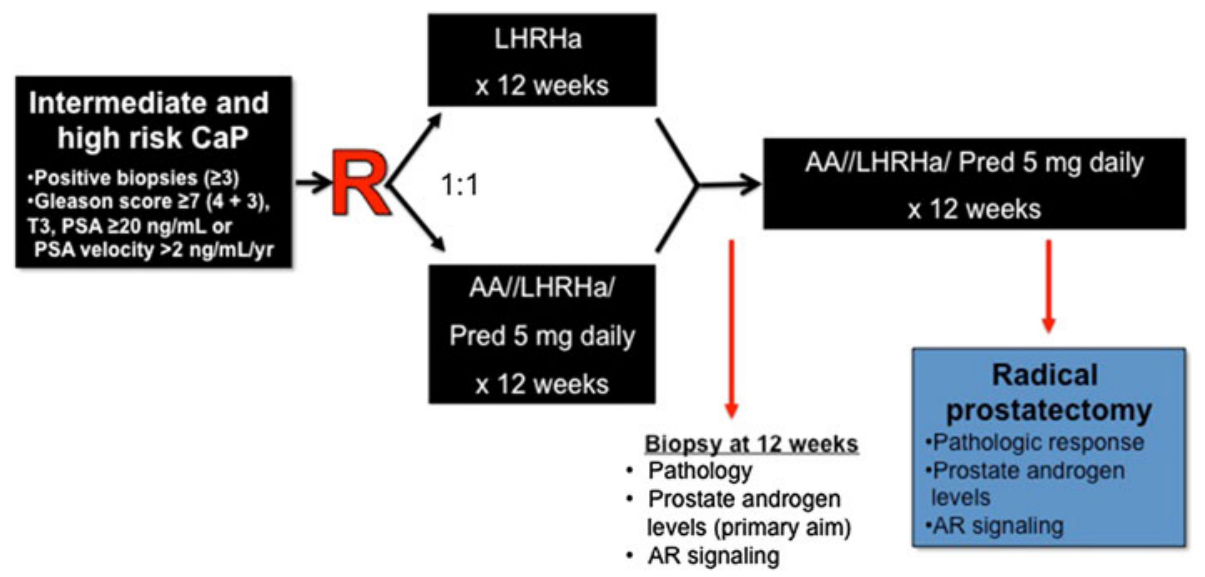

Fig. 2 Randomized, phase II neoadjuvant study of abiraterone acetate, leuprolide, and prednisone in newly diagnosed intermediate and high-risk patients with prostate cancer undergoing radical prostatectomy $(N=58)$. $A A$

pharmacodynamic effects in high-risk patients fit for prostatectomy [56]. In this multiinstitutional, phase II study, patients were initially randomized to leuprolide or leuprolide/abiraterone acetate/prednisone (5 mg daily) for 3 months (Fig. 2) [56]. The primary endpoint of the trial was the evaluation of prostate testosterone and DHT levels after 3 months of therapy. After the first 3 months of therapy, all patients were treated with an additional 3 months of leuprolide/ abiraterone acetate/prednisone (5 mg daily). Thus, one cohort received 6 months of abiraterone acetate and the other group received 3 months of abiraterone acetate, but all patients received 6 months of ADT in total [56]. All participants had prostatectomy after 6 months of ADT. Eligibility targeted high-risk patients and required that patients had positive biopsies in three or more cores, Gleason score $\geq 7(4+3)$, stage T3 disease, PSA $\geq 20 \mathrm{ng} / \mathrm{mL}$, or a PSA velocity of $>2 \mathrm{ng}$ / $\mathrm{mL} /$ year [56]. Fifty-eight patients were accrued over 18 months. Secondary endpoints included pathologic staging at prostatectomy, PSA response, and assessment of androgen abiraterone acetate, $A R$ androgen receptor, $C a P$ prostate cancer, $\mathrm{LHRHa}$ leuprolide acetate, Pred prednisone, PSA prostate-specific antigen, $q d$ once daily, $R$ randomize

receptor signaling. Publication of the results of this work is pending, and only data presented at the 2012 American Society of Clinical Oncology (ASCO) Annual Meeting is described here [56].

Longer (6-month) compared with shorter (3-month) abiraterone acetate administration achieved a greater percentage of pathologic complete response (CR) $(10 \%$ vs. $4 \%$, respectively), near pathologic CR ( $24 \%$ vs. $11 \%$, respectively), and any disease shrinkage, as assessed by a composite endpoint of true pathologic CR plus near CR ( $15 \%$ vs. $34 \%$, respectively) [56]. The primary endpoint was to induce a significant reduction in prostate tumor androgen levels (DHT and DHEA) at the 12-week mark and was realized with the combination therapy compared to leuprolide alone $(P<0.0001)$. In the group who started abiraterone acetate after 12 weeks of leuprolide monotherapy, the addition of abiraterone acetate induced a statistically significant decrease in DHT and DHEA levels at 24 weeks compared to their pre-abiraterone acetate baseline at 12 weeks $(P=0.0004)$ [56]. Similar decreases were seen with 
androstenedione and, to a lesser degree, with testosterone. As expected, given the inhibition of CYP17 $\alpha$-hydroxylase conversion of pregnenolone and progesterone to their $17-\alpha$ hydroxy derivatives, pregnenolone and progesterone levels were higher in prostate tissue from abiraterone acetate-treated patients as compared to the corresponding levels from patients in the leuprolide monotherapy arm at 12 weeks [56].

The safety of neoadjuvant abiraterone acetate administration is paramount given that patients are generally asymptomatic with excellent quality of life. No new safety signals were identified during this study and the toxicity profile was consistent with past phase III data in the chemotherapy-refractory setting $[4,56]$. Grade 3 liver function abnormalities did not increase significantly with the longer duration of dosing: $7.1 \%$ vs. $10 \%$ with 12 - and 24-week dosing of abiraterone acetate, respectively [56].

Past trials of ADT with an LHRH agonist administered neoadjuvant to prostatectomy did not alter the PSA relapse rate and this approach has been abandoned from clinical practice [57, 58]. The current availability of more effective ADT has renewed interest in the analysis of combined systemic androgen deprivation with surgery to increase cure rates in patients at high risk of recurrence. At the Dana-Farber Cancer Institute in the US, subsequent neoadjuvant trials have been completed or are planned, evaluating intense ADT neoadjuvant to prostatectomy, assessing various compounds, including combinations of abiraterone acetate, enzalutamide, dutasteride, and ARN509. We hope to determine the optimal combination and duration of neoadjuvant ADT that will lead to a randomized, phase III trial in high-risk patients.

\section{Resistance Mechanisms in the AR Pathway}

As with all available therapies, eventual resistance to abiraterone acetate occurs. Possible mechanisms of resistance include either reactivation of the $\mathrm{AR}$ or AR-independent mechanisms [22, 27]. Nelson described four molecular states for AR activation in prostate cancer: (1) endocrine androgen dependent and AR dependent; (2) intracrine androgen and AR dependent; (3) androgen (ligand) independent and AR dependent; and (4) ligand and AR independent [22]. With its ability to inhibit both host and tumoral testosterone production, abiraterone acetate seems an ideal treatment for ligand-dependent disease. But primary or eventual on-therapy resistance ultimately occurs, likely due to selective pressure from ongoing therapy, which can lead to transformation to a ligand-independent state [22]. Alternative ligand-dependent mechanisms include altered steroid biosynthesis in the tumor microenvironment, increased 'backdoor' synthesis of DHT, and circumvention of testosterone as the precursor [31, 54]. In the first phase I study of abiraterone acetate where no concomitant steroids were given, increased presence of steroids upstream of CYP17 that continued to drive AR signaling were a purported source of resistance. The addition of steroids in these patients temporarily reversed progression in 4 of 15 patients [32].

A rising PSA often heralds progression on abiraterone acetate. When this change occurs in the setting of undetectable serum and tissue androgens, it suggests persistent dependence on AR signaling but ligand independence (Nelson disease state 3) [22]. This transformation may be driven by cross-talk with other signaling pathways that can activate AR signaling in the absence of androgens, and AR splice variants that 
create constitutively active ARs [22, 59, 60]. Possible ligand-independent mechanisms that drive progression during treatment with androgen biosynthesis inhibitors could include loss of phosphatase and tensin homolog on chromosome 10 (PTEN) loss, overexpression of factors in the phosphatidylinositide 3-kinase/ protein kinase B (PI3-kinase/Akt) pathway, overexpression of Bcl-2, c-Met, and others [54, 61-67].

Transformation to complete independence from both androgen ligand and signaling (Nelson disease state 4) is rare but should be considered when PSA remains undetectable on abiraterone acetate but the patient is clearly progressing according to scans or symptoms. We recommend treatment at this juncture with novel, investigational, non-androgen-directed strategies, such as chemotherapy. Conversely, Nelson disease states 2 and 3 imply that androgens or AR signaling continue to drive tumor growth and thus, the tumors may remain sensitive to further hormonal interventions.

\section{FUTURE DIRECTIONS}

Several innovative therapeutic strategies incorporating abiraterone acetate are under investigation or in development. Abiraterone acetate's safety profile makes it well suited to combine with other agents in order to increase its efficacy and target resistance pathways. Additionally, its tolerability makes it an ideal candidate to consider earlier in the course of treatment, when patients are asymptomatic, such as in the neoadjuvant, biochemical recurrence (D0), hormone-naïve, or prechemotherapy metastatic settings.

The recent proof of abiraterone acetate's activity and safety in the chemotherapy-naïve, metastatic CRPC setting imparts confidence for its safe investigation in earlier disease states. The phase II Impact of Abiraterone Acetate in Prostate-Specific Antigen (IMAAGEN) study will explore its ability to effectively decrease PSA in 125 patients with biochemical progression on GnRH monotherapy but with no evidence of metastasis (ClinicalTrials.gov identifier: NCT01314118). This disease state is an area of unmet need where no standard therapy has demonstrated a survival benefit.

One mechanism of persistent AR signaling in CRPC is increased expression of enzymes, such as AKR1C3 and SRD5A1, which are critical in the regulation of testosterone and DHT synthesis from precursor steroids [28]. Enzymes, such as SRD5A1, may lead to tumor escape from CYP17 inhibition and GnRH axis blockade. We recently completed a phase II study in metastatic CPRC investigating the addition of dutasteride, a dual SRD5A1/ SRD5A2 inhibitor, to abiraterone acetate (ClinicalTrials.gov\# NCT01393730). Prior chemotherapy or any number of hormonal therapies was permitted, but patients were required to have a metastatic site amenable to biopsy pre-treatment and at progression. The primary objective was to analyze possible ARrelated mechanisms of abiraterone acetate resistance. Clinical outcomes data are maturing, and ongoing analysis of the tumor specimens obtained prior to treatment and at progression will evaluate the AR genetic sequence (e.g., mutations, splice variants), ARregulated gene expression, tumor androgen levels, and profiling of enzymes involved in androgen synthesis and metabolism.

The AR antagonist and signaling inhibitor, enzalutamide, is another avenue by which we can target persistent AR signaling. Because enzalutamide does not inhibit androgen production, combining it with the potent testosterone synthesis inhibitor, abiraterone 
acetate, makes sense in order to target all levels of androgen production and signaling [68]. Leveraging their non-overlapping and potentially synergistic effects, emerging studies will assess the combinations of enzalutamide or ARN509, an AR antagonist currently in development, and abiraterone or TAK-700, a CYP17 inhibitor currently in development, in a variety of disease settings. Follow-up studies to our abiraterone/leuprolide neoadjuvant trial are planned in which we will escalate the intensity of androgen deprivation and co-pathway targeting. Within the United States Alliance Clinical Trials co-operative group, a trial of 'androgen annihilation' for men with biochemical relapse is in development. Additionally, a co-operative group phase III trial will randomize patients to enzalutamide plus abiraterone acetate versus enzalutamide monotherapy in metastatic CRPC patients prior to chemotherapy (M. Morris, Memorial SloanKettering Cancer Center: personal communication, Alliance meeting November 2012) [69].

The survival advantage of sipuleucel-T has considerably challenged the dogma that prostate cancer is not an immunotherapysensitive tumor. Nesslinger and colleagues [70] demonstrated that hormone therapy and radiation could elicit antigen-specific immune responses in the form of development of autoantibodies to tumor-associated antigens. Further, as reviewed by Aragon-Ching and colleagues [71], there is evidence that ADT can augment lymphopoiesis and enhance immune responses to vaccines, restore thymopoiesis, increase B cell development, and reverse tolerance to prostate cancer antigens. Thus, augmenting tertiary hormone approaches, such as abiraterone, with immunomodulatory agents such as sipuleucel-T is rational and the source of ongoing investigation. Aiming to capitalize on the survival benefit of both abiraterone and sipuleucel-T, and provide patients with a tangible positive effect in terms of response and PFS, a randomized phase II study is underway investigating concurrent versus sequential use of abiraterone acetate/prednisone and sipuleucel-T (ClinicalTrials.gov identifier: NCT01431391). A relevant question with this combination is whether the concomitant steroids given as standard to prevent abiraterone acetateinduced ME will dampen the immune response to sipuleucel-T. Data presented at ASCO 2013 suggested that steroids did not diminish the immune system response to sipuleucel-T, but this conclusion awaits validation [72].

Another mechanism of resistance may be upregulation of programmed death 1 ligand (PD-L1) induced by androgen blockade (C. Drake, Johns Hopkins University: personal communication). Ongoing studies are evaluating the mechanism and timing of PDL1 upregulation in response to androgen ablation. Combination therapy directed at targeting both hormonal dependence with agents, such as abiraterone acetate, and resistance pathways, such as PD-1 or cytotoxic T-lymphocyte antigen-4 (CTLA-4), could be synergistic. A trial evaluating abiraterone acetate with CTLA-blockade with ipilimumab is ongoing in CRPC (ClinicalTrials.gov identifier: NCT01688492).

Finally, as several of the prior studies have shown, approximately $25 \%$ of patients have primary abiraterone acetate resistance, highlighting the need for the identification of predictive markers and signatures that can better characterize these patients a priori, and direct them to more rational therapeutics or clinical trials. Efforts to counteract resistance mechanisms to abiraterone include 
combination strategies with c-MET blockade, heat shock protein inhibitors, or histone deacetylase inhibitors aimed at disrupting the AR transcription complex. For example, Sweeney and colleagues at the Dana-Farber Cancer Institute are evaluating abiraterone acetate in combination with cabozantinib, an oral c-Met and vascular endothelial growth factor receptor multi-tyrosine kinase inhibitor (ClinicalTrials.gov \# NCT01574937), an agent, which has shown promising and unexpected efficacy in bony metastases, as well as combination therapy with OGX-427 at abiraterone progression (ClinicalTrials.gov \#NCT01681433).

\section{CONCLUSION}

The last several years have engendered tremendous optimism for the treatment of advanced prostate cancer. Patients now have access to more than five therapies that prolong survival, with several more on the horizon. These much-needed advances in survival are perhaps best demonstrated by the placebo arm of the chemotherapy-naïve, phase III abiraterone acetate trial, where patients had a median OS of 27 months, an increase from 18 months in the docetaxel era $[9,17]$. While both cohorts of patients received steroids, which have some activity, and there has also likely been an inherent shift to initiating therapy earlier, this approximately 10-month improvement in median OS is more likely the consequence of the increased availability and sequential use of multiple survival-improving therapies, which patients on these trials had access to after unblinding [9]. Tolerable, effective agents, such as abiraterone acetate, are critical for improving survival while maintaining quality of life. Abiraterone acetate remains a valuable option post-chemotherapy, but the FDA's expanded indication to the chemotherapy-naïve setting addresses a critical unmet need, and in the future, abiraterone acetate is likely to be used predominantly prior to chemotherapy. Abiraterone acetate delivers clinically meaningful advantages in its oral administration, favorable tolerability profile, and high efficacy. Further optimization of its use is being investigated with the use of lower doses or no concurrent steroids, and without concomitant gonadal suppression.

In addition to abiraterone acetate's clinical benefits, its development is noteworthy for having confirmed the clinical importance of intratumoral androgen synthesis, which may promote therapeutic resistance, and also for validating the principle that $\mathrm{AR}$ signaling continues to drive disease progression after the development of castration resistance $[4,9,25]$. Progress will require the identification of predictive biomarkers and the development of combination therapies that will enhance abiraterone acetate's efficacy and thwart resistance pathways. Future study will address whether its use concurrently or in sequence with other highly effective agents, such as enzalutamide or sipuleucel-T, will result in synergistic effects, and whether, as is currently done with leuprolide acetate, abiraterone acetate should be continued past progression.

\section{ACKNOWLEDGMENTS}

This work was supported in part by the Fairweather and Uribe Prostate Cancer Research Funds. Drs. Harshman and Taplin contributed equally to the original design, writing, and revision of this review article. No 
editorial or medical writing assistance was utilized. Prior to peer review, Janssen was offered the opportunity to review this paper for scientific accuracy. Dr. Harshman is the guarantor for this article, and takes responsibility for the integrity of the work as a whole.

Conflict of interest. Lauren C. Harshman declares no conflict of interest. Mary-Ellen Taplin has received research funding and has taken part in an advisory board for Johnson \& Johnson.

Open Access. This article is distributed under the terms of the Creative Commons Attribution Noncommercial License which permits any noncommercial use, distribution, and reproduction in any medium, provided the original author(s) and the source are credited.

\section{REFERENCES}

1. Siegel R, Naishadham D, Jemal A. Cancer statistics, 2012. CA Cancer J Clin. 2012;62(10):29.

2. de Bono JS, Oudard S, Ozguroglu $\mathrm{M}$, et al. Prednisone plus cabazitaxel or mitoxantrone for metastatic castration-resistant prostate cancer progressing after docetaxel treatment: a randomised open-label trial. Lancet. 2010; 376(1147):54.

3. Kantoff PW, Higano CS, Shore ND, et al. Sipuleucel$\mathrm{T}$ immunotherapy for castration-resistant prostate cancer. N Engl J Med. 2010;363(411):22.

4. de Bono JS, Logothetis CJ, Molina A, et al. Abiraterone and increased survival in metastatic prostate cancer. N Engl J Med. 1995; 2011(364):2005.

5. Fizazi K, Scher HI, Molina A, et al. Abiraterone acetate for treatment of metastatic castrationresistant prostate cancer: final overall survival analysis of the COU-AA-301 randomised, doubleblind, placebo-controlled phase 3 study. Lancet Oncol. 2012;13(983):92.
6. Scher HI, Fizazi K, Saad F, et al. Increased survival with enzalutamide in prostate cancer after chemotherapy. N Engl J Med. 2012;367(1187):97.

7. Sartor AO, Heinrich D, Helle SI, et al. Radium-223 chloride impact on skeletal-related events in patients with castration-resistant prostate cancer (CRPC) with bone metastases: a phase III randomized trial (ALSYMPCA). J Clin Oncol 2012 Genitourinary Cancers Symposium. 2012;30(Suppl.): Abstract 9.

8. Parker C, Heinrich D, O'Sullivan JM, et al. Overall survival benefit and safety profile of radium-223 chloride, a first-in-class alpha-pharmaceutical: results from a phase III randomized trial (ALSYMPCA) in patients with castration-resistant prostate cancer (CRPC) with bone metastases. J Clin Oncol. Genitourinary Cancers Symposium. 2012;30(Suppl.): Abstract 8.

9. Ryan CJ, Smith MR, de Bono JS, et al. Abiraterone in metastatic prostate cancer without previous chemotherapy. N Engl J Med. 2013;368(138):48.

10. Fizazi K, Carducci M, Smith M, et al. Denosumab versus zoledronic acid for treatment of bone metastases in men with castration-resistant prostate cancer: a randomised, double-blind study. Lancet. 2011;377(813):22.

11. Smith MR, Saad F, Coleman R, et al. Denosumab and bone-metastasis-free survival in men with castration-resistant prostate cancer: results of a phase 3, randomised, placebo-controlled trial. Lancet. 2012;379(39):46.

12. Balk SP. Androgen receptor as a target in androgenindependent prostate cancer. Urology. 2002;60:132-8, discussion 89.

13. Gelmann EP. Molecular biology of the androgen receptor. J Clin Oncol. 2002;20(3001):15.

14. Huggins C, Stevens RE, Hodges CV. Studies on prostatic cancer. II. The effects of castration on advanced carcinoma of the prostate gland. Arch Surg. 1941;43(209):23.

15. Crawford ED, Eisenberger MA, McLeod DG, et al. A controlled trial of leuprolide with and without flutamide in prostatic carcinoma. $\mathrm{N}$ Engl J Med. 1989;321:419-24.

16. Samson DJ, Seidenfeld J, Schmitt B, et al. Systematic review and meta-analysis of monotherapy compared with combined androgen blockade for patients with advanced prostate carcinoma. Cancer. 2002;95:361-76.

17. Tannock IF, de Wit R, Berry WR, et al. Docetaxel plus prednisone or mitoxantrone plus prednisone 
for advanced prostate cancer. $\mathrm{N}$ Engl $\mathrm{J}$ Med. 2004;351(1502):12.

18. Davis NB, Ryan CW, Stadler WM, Vogelzang NJ. A phase II study of nilutamide in men with prostate cancer after the failure of flutamide or bicalutamide therapy. BJU Int. 2005;96(787):90.

19. Desai A, Stadler WM, Vogelzang NJ. Nilutamide: possible utility as a second-line hormonal agent. Urology. 1016;2001(58):20.

20. Nakabayashi M, Regan MM, Lifsey D, et al. Efficacy of nilutamide as secondary hormonal therapy in androgen-independent prostate cancer. BJU Int. 2005;96(783):6.

21. Small EJ, Halabi S, Dawson NA, et al. Antiandrogen withdrawal alone or in combination with ketoconazole in androgen-independent prostate cancer patients: a phase III trial (CALGB 9583). J Clin Oncol. 1025;2004(22):33.

22. Nelson PS. Molecular states underlying androgen receptor activation: a framework for therapeutics targeting androgen signaling in prostate cancer. J Clin Oncol. 2012;30(644):6.

23. Attard G, Belldegrun AS, de Bono JS. Selective blockade of androgenic steroid synthesis by novel lyase inhibitors as a therapeutic strategy for treating metastatic prostate cancer. BJU Int. 2005;96:1241-6.

24. Page ST, Lin DW, Mostaghel EA, et al. Persistent intraprostatic androgen concentrations after medical castration in healthy men. J Clin Endocrinol Metab. 2006;91(3850):6.

25. Mostaghel EA, Page ST, Lin DW, et al. Intraprostatic androgens and androgen-regulated gene expression persist after testosterone suppression: therapeutic implications for castration-resistant prostate cancer. Cancer Res. 2007;67(5033):41.

26. Fenton MA, Shuster TD, Fertig AM, et al. Functional characterization of mutant androgen receptors from androgen-independent prostate cancer. Clin Cancer Res. 1997;3(1383):8.

27. Massard C, Fizazi K. Targeting continued androgen receptor signaling in prostate cancer. Clin Canc Res. 2011;17(3876):83.

28. Stanbrough M, Bubley GJ, Ross K, et al. Increased expression of genes converting adrenal androgens to testosterone in androgen-independent prostate cancer. Cancer Res. 2006;66(2815):25.

29. Sun S, Sprenger CC, Vessella RL, et al. Castration resistance in human prostate cancer is conferred by a frequently occurring androgen receptor splice variant. J Clin Investig. 2010;120(2715):30.

30. Taplin ME, Bubley GJ, Shuster TD, et al. Mutation of the androgen-receptor gene in metastatic androgen-independent prostate cancer. N Engl J Med. 1995;332(1393):8.

31. Chang KH, Li R, Papari-Zareei $\mathrm{M}$, et al. Dihydrotestosterone synthesis bypasses testosterone to drive castration-resistant prostate cancer. Proc Natl Acad Sci USA. 2011;108(13728):33.

32. Attard G, Reid AH, A'Hern R, et al. Selective inhibition of CYP17 with abiraterone acetate is highly active in the treatment of castrationresistant prostate cancer. J Clin Oncol. 2009; 27(3742):8

33. Cleutjens KB, van der Korput HA, van Eekelen CC, van Rooij HC, Faber PW, Trapman J. An androgen response element in a far upstream enhancer region is essential for high, androgen-regulated activity of the prostate-specific antigen promoter. Mol Endocrinol. 1997;11(148):61.

34. Riegman PH, Vlietstra RJ, van der Korput JA, Brinkmann AO, Trapman J. The promoter of the prostate-specific antigen gene contains a functional androgen responsive element. Mol Endocrinol. 1921;1991(5):30.

35. Joyce R, Fenton MA, Rode P, et al. High dose bicalutamide for androgen independent prostate cancer: effect of prior hormonal therapy. J Urol. 1998;159(149):53.

36. Taplin ME, Regan MM, Ko YJ, et al. Phase II study of androgen synthesis inhibition with ketoconazole, hydrocortisone, and dutasteride in asymptomatic castration-resistant prostate cancer. Clin Cancer Res. 2009;15(7099):105.

37. Potter GA, Barrie SE, Jarman M, Rowlands MG. Novel steroidal inhibitors of human cytochrome P45017 alpha (17 alpha-hydroxylase-C17,20-lyase): potential agents for the treatment of prostatic cancer. J Med Chem. 1995;38(2463):71.

38. Attard G, Richards J, de Bono JS. New strategies in metastatic prostate cancer: targeting the androgen receptor signaling pathway. Clin Cancer Res. 2011;17(1649):57.

39. Attard G, Reid AH, Yap TA, et al. Phase I clinical trial of a selective inhibitor of CYP17, abiraterone acetate, confirms that castration-resistant prostate cancer commonly remains hormone driven. J Clin Oncol. 2008;26(4563):71.

40. Ryan CJ, Shah S, Efstathiou E, et al. Phase II study of abiraterone acetate in chemotherapy-naive 
metastatic castration-resistant prostate cancer displaying bone flare discordant with serologic response. Clin Cancer Res. 2011;17(4854):61.

41. Ryan CJ, Smith MR, Fong L, et al. Phase I clinical trial of the CYP17 inhibitor abiraterone acetate demonstrating clinical activity in patients with castration-resistant prostate cancer who received prior ketoconazole therapy. J Clin Oncol. 2010;28(1481):8.

42. O'Donnell A, Judson I, Dowsett M, et al. Hormonal impact of the 17 alpha-hydroxylase/C $(17,20)$-lyase inhibitor abiraterone acetate (CB7630) in patients with prostate cancer. Br J Cancer. 2004;90(2317):25.

43. Grigoryev DN, Long BJ, Njar VC, Brodie AH. Pregnenolone stimulates LNCaP prostate cancer cell growth via the mutated androgen receptor. J Steroid Biochem Mol Biol. 2000;75(1):10.

44. Taplin ME, Rajeshkumar B, Halabi S, et al. Androgen receptor mutations in androgenindependent prostate cancer: Cancer and Leukemia Group B Study 9663. J Clin Oncol. 2003;21(2673):8.

45. Danila DC, Morris MJ, de Bono JS, et al. Phase II multicenter study of abiraterone acetate plus prednisone therapy in patients with docetaxeltreated castration-resistant prostate cancer. J Clin Oncol. 2010;28(1496):501.

46. Reid AH, Attard G, Danila DC, et al. Significant and sustained antitumor activity in post-docetaxel, castration-resistant prostate cancer with the CYP17 inhibitor abiraterone acetate. J Clin Oncol. 2010;28(1489):95.

47. Sternberg CN, Molina A, North S, et al. Effect of abiraterone acetate on fatigue in patients with metastatic castration-resistant prostate cancer after docetaxel chemotherapy. Ann Oncol. 1017; 2013(24):25.

48. Logothetis CJ, Basch E, Molina A, et al. Effect of abiraterone acetate and prednisone compared with placebo and prednisone on pain control and skeletal-related events in patients with metastatic castration-resistant prostate cancer: exploratory analysis of data from the COU-AA-301 randomised trial. Lancet Oncol. 2012;13(1210):7.

49. Scher HI, Halabi S, Tannock I, et al. Design and end points of clinical trials for patients with progressive prostate cancer and castrate levels of testosterone: recommendations of the Prostate Cancer Clinical Trials Working Group. J Clin Oncol. 2008; 26(1148):59.

50. Kantoff PW, Halabi S, Conaway $M$, et al. Hydrocortisone with or without mitoxantrone in men with hormone-refractory prostate cancer: results of the cancer and leukemia group B 9182 study. J Clin Oncol. 1999;17(2506):13.

51. Tannock IF, Osoba D, Stockler MR, et al. Chemotherapy with mitoxantrone plus prednisone or prednisone alone for symptomatic hormone-resistant prostate cancer: a Canadian randomized trial with palliative end points. J Clin Oncol. 1996;14(1756):64.

52. Ryan CJ, Smith MR, et al. Interim analysis (IA) results of COU-AA-302, a randomized, phase III study of abiraterone acetate (AA) in chemotherapynaive patients (pts) with metastatic castrationresistant prostate cancer (mCRPC). J Clin Oncol. 2012;30(Suppl.): Abstract LBA4518.

53. US Food and Drug Administration. Abiraterone acetate. http://www.fda.gov/Drugs/InformationOn Drugs/ApprovedDrugs/ucm331628.htm. Accessed 12 December 2012.

54. Efstathiou E, Titus M, Tsavachidou D, et al. Effects of abiraterone acetate on androgen signaling in castrate-resistant prostate cancer in bone. J Clin Oncol. 2012;30(637):43.

55. Albertsen PC. The face of high risk prostate cancer. World J Urol. 2008;26:205-10.

56. Taplin ME, Montgomery RB, Logothetis C, et al. Effect of neoadjuvant abiraterone acetate (AA) plus leuprolide acetate (LHRHa) on PSA, pathological complete response (pCR), and near pCR in localized high-risk prostate cancer (LHRPC): results of a randomized phase II study. J Clin Oncol. 2012;30(Suppl.): Abstract 4521.

57. Gleave ME, Goldenberg SL, Chin JL, et al. Randomized comparative study of 3 versus 8-month neoadjuvant hormonal therapy before radical prostatectomy: biochemical and pathological effects. J Urol. 2001;166:500-6, discussion 67 .

58. Gleave ME, La Bianca SE, Goldenberg SL, Jones EC, Bruchovsky N, Sullivan LD. Long-term neoadjuvant hormone therapy prior to radical prostatectomy: evaluation of risk for biochemical recurrence at 5-year follow-up. Urology. 2000;56(289):94.

59. Guo Z, Yang X, Sun F, et al. A novel androgen receptor splice variant is up-regulated during prostate cancer progression and promotes androgen depletion-resistant growth. Cancer Res. 2009;69(2305):13.

60. Hu R, Dunn TA, Wei S, et al. Ligand-independent androgen receptor variants derived from splicing of cryptic exons signify hormone-refractory prostate cancer. Cancer Res. 2009;69(16):22. 
61. Cairns P, Okami K, Halachmi S, et al. Frequent inactivation of PTEN/MMAC1 in primary prostate cancer. Cancer Res. 1997;57(4997):5000.

62. Knudsen BS, Edlund M. Prostate cancer and the met hepatocyte growth factor receptor. Adv Cancer Res. 2004;91(31):67.

63. Knudsen BS, Gmyrek GA, Inra J, et al. High expression of the Met receptor in prostate cancer metastasis to bone. Urology. 2002;60(1113):7.

64. McMenamin ME, Soung P, Perera S, Kaplan I, Loda $M$, Sellers WR. Loss of PTEN expression in paraffinembedded primary prostate cancer correlates with high Gleason score and advanced stage. Cancer Res. 1999;59(4291):6.

65. Tu WH, Zhu C, Clark C, Christensen JG, Sun Z. Efficacy of c-Met inhibitor for advanced prostate cancer. BMC Cancer. 2010;10:556.

66. Verras M, Lee J, Xue H, Li TH, Wang Y, Sun Z. The androgen receptor negatively regulates the expression of c-Met: implications for a novel mechanism of prostate cancer progression. Cancer Res. 2007;67(967):75.

67. Watanabe $M$, Fukutome $\mathrm{K}$, Kato $\mathrm{H}$, et al. Progression-linked overexpression of c-Met in prostatic intraepithelial neoplasia and latent as well as clinical prostate cancers. Cancer Lett. 1999;141(173):8.

68. Tran C, Ouk S, Clegg NJ, et al. Development of a second-generation antiandrogen for treatment of advanced prostate cancer. Science. 2009;324(787):90.

69. Vogelzang NJ. Enzalutamide-a major advance in the treatment of metastatic prostate cancer. N Eng J Med. 2012;367(1256):7.

70. Nesslinger NJ, Sahota RA, Stone B, et al. Standard treatments induce antigen-specific immune responses in prostate cancer. Clin Cancer Res. 2007;13(1493):502.

71. Aragon-Ching JB, Williams KM, Gulley JL. Impact of androgen-deprivation therapy on the immune system: implications for combination therapy of prostate cancer. Front Biosci. 2007;12(4957):71.

72. Small EJ, Lance RS, Redfern $\mathrm{CH}$, et al. A randomized phase II trial of sipuleucel-T with concurrent or sequential abiraterone acetate (AA) plus prednisone (P) in metastatic castrate-resistant prostate cancer (mCRPC). J Clin Oncol. 2013;31(Suppl.): Abstract 5047. 